\title{
Gut-Liver Interactions: Full of New Excitements
}

Whereas the genetic background of many inflammatory gastrointestinal disorders highlights an important role for immunity, current evidence has introduced the gut microbiota as a new key player. Only recently investigators have started to understand the important relationship between the genome and microbiome. Chu et al. [1] recently tested Bacteroides fragilis, a known beneficial bacterium, by treating mice that had nonfunctional mutations in the 2 well-known Crohn's disease risk factor genes, NOD2 and ATG16L1. If one of these genes were defective, the mice were unable to develop a protective immune response in response to Bacteroides fragilis. Importantly, even cells from patients who exhibited the mutated version of these genes showed no anti-inflammatory response to Bacteroides fragilis. This exciting study demonstrates the interaction between immunity and the microbiome, and furthermore suggests that certain designed probiotics might not work in some patient populations. In this sense, it is fascinating to see that the microbiota also might play a fundamental role in many liver diseases such as primary sclerosing cholangitis (PSC) and others [2]. In PSC, a prototypic disease directed by immunity, besides the gut microbiota also 'bile microbiota/ pathobionts' could play a role. We have to keep in mind, that the interaction between gut and liver disorders is not only 'one-directional' but rather 'bi-directional' as we have recently acknowledged that bile acids are probably one of the major confounders of the gut microbiota and thereby might affect many diseases throughout the gastrointestinal tract [3]. These studies highlight that for a better understanding of various diseases, a holistic approach is needed and the field microbiota \pm innate/adaptive immunity definitely has 'tightened' the gut-liver axis in the past years. Therefore, the microbiota might reflect the unifying force in the pathophysiology of many gastrointestinal and liver disorders.

For these reasons, the Falk symposium 201 has been initiated to specifically address gut-liver interactions covering diseases from inflammatory bowel diseases (IBD) to nonalcoholic steatohepatitis. This symposium intended to stimulate attendants to discuss gut-liver interactions from pathophysiology toward therapeutic approaches. We had been lucky in attracting excellent and well-renowned speakers from both the gastroenterology and hepatology fields. The selection of topics was based on the assumption to discuss fields where such interactions are obvious and highly relevant as in nonalcoholic fatty liver disease (NAFLD) or IBD. An important feature of this symposium was the role of innate immunity and gut microbiota in those interactions, and these aspects were es-

\section{KARGER}

E-Mail karger@karger.com

www.karger.com/ddi
(C) 2016 S. Karger AG, Basel

0257-2753/16/0345-0001\$39.50/0 
pecially highlighted. Colleagues with a primarily clinical focus received a thorough update in topics such as IBD, NAFLD, alcoholic liver disease, primary biliary cholangitis or PSC. In many countries, clinical care of liver and gastrointestinal patients is separated because of various reasons including healthcare structure, science and new research avenues might bring together these close areas of medicine in the near future. It is a better understanding of pathophysiological interactions between the liver and the gastrointestinal tract that might advance and improve patient care in the future.

H. Tilg, Innsbruck

\section{References}

1 Chu H, Khosravi A, Kusumawardhani IP, Kwon AH, Vasconcelos AC, Cunha LD, et al: Gene-microbiota interactions contribute to the pathogenesis of inflammatory bowel disease. Science 2016;352:1116-1120.
2 Kummen M, Holm K, Anmarkrud JA, Nygard S, Vesterhus M, Hoivik ML, et al: The gut microbial profile in patients with primary sclerosing cholangitis is distinct from patients with ulcerative colitis without biliary disease and healthy controls. Gut 2016; pii:gutjnl2015-310500.
3 Devkota S, Chang EB: Interactions between diet, bile acid metabolism, gut microbiota, and inflammatory bowel diseases. Dig Dis 2015;33:351-356. 